\title{
CIRCUITS INTÉGRÉS \\ photoniques silicium
}

\author{
Laurent VIVIEN ${ }^{1}$, Delphine MARRIS-MORINI ${ }^{1}$, Eric CASSAN $^{1}$, Carlos ALONSO-RAMOS ${ }^{1}$, Charles BAUDOT ${ }^{2}$, \\ Frédéric BCEUF ${ }^{2}$, Bertrand SZELAG ${ }^{3}$, \\ ${ }^{1}$ Centre de Nanosciences et Nanotechnologies (C2N), CNRS, Université Paris Sud, Université Paris Saclay, UMR9001, 91400 Orsay \\ ${ }^{2}$ STMicroelectronics SAS, 850 rue Jean Monnet, 38920 Crolles \\ ${ }^{3}$ CEA, LETI, Minatec Campus, 17 rue des Martyrs, 38054 Grenoble \\ laurent.vivien@c2n.upsaclay.fr
}

La photonique silicium suit la devise : "plus petit, moins cher, plus rapide», comme la microélectronique plusieurs années auparavant, en exploitant une intégration à très grande échelle des composants et circuits intégrés de plus en plus complexes. L'incroyable évolution des systèmes communicants avec en particulier le déploiement des réseaux Internet et mobiles, des objets connectés et des capteurs a fait émerger la photonique silicium pour répondre à ces nouveaux enjeux majeurs.

\section{I}

e silicium (Si) est l'un des matériaux qui ont radicalement technologique et sociétal à partir de l'invention du transistor en 1947. L'extraordinaire évolution vers la miniaturisation des transistors et l'augmentation de leur nombre pour la réalisation de circuits intégrés et fonctions complexes ont conduit au développement de l'industrie de la microélectronique et de ses multiples applications résultantes. Cette évolution a principalement suivi la «loi de Moore», énoncée par Gordon Moore en 1965, prévoyant une augmentation exponentielle du nombre de transistors par puce tous les 18 mois. Depuis cette époque, le silicium est le matériau le plus utilisé de $l^{\prime}$ industrie des semi-conducteurs pour des raisons physiques et technologiques (qualité de son oxyde, la silice, et de l'interface avec son oxyde, propriétés mécaniques et thermiques) et économiques (large accès à la matière première, le silicium ; retour sur investissement des infrastructures rapidement atteint). Cependant, depuis quelques années, les progrès fulgurants de l'électronique silicium atteignent des limites, en particulier en termes de puissance consommée bien trop élevée dans une ère où l'impact énergétique est une priorité majeure, et de bande passante pour laquelle les débits visés pour répondre à la demande croissante devient trop faible. En effet, les nombreuses interconnexions électriques nécessaires aux transferts de données au sein des puces électroniques sont une source majeure de dissipation de puissance. Pour pallier ces limitations, les interconnexions optiques, consistant à miniaturiser des liens optiques complets en vue de véhiculer des signaux rapides sur puce, sont considérées depuis une vingtaine d'années comme une solution alternative intéressante au tout-électronique.

Afin de conserver la compatibilité avec les technologies de la microélectronique (essentiellement CMOS), l'utilisation du silicium pour la photonique a rapidement été considérée comme la voie principale à explorer, laissant de côté des solutions telles que l'intégration totalement hybride de composants optoélectroniques à base de semiconducteurs III/V déjà développées pour le domaine des télécoms par fibres optiques. L'évolution vers la photonique Si a été notamment permise grâce à l'émergence de substrats polyvalents en silicium sur isolant (SOI) et aux propriétés optiques intéressantes du silicium offrant une large fenêtre de transparence pour des longueurs d'onde allant de $1,1 \mu \mathrm{m}$ à $7 \mu \mathrm{m}$ [2]. En outre, les substrats SOI donnent accès à un fort contraste d'indice de réfraction entre celui du silicium $\operatorname{Si}\left(n_{S i} \approx 3,47\right.$ vers $\lambda=1,55 \mu \mathrm{m})$ et celui de la silice $\mathrm{SiO}_{2}$ $\left(n_{\mathrm{SiO} 2} \approx 1,45\right)$, permettant le développement de composants compacts adaptés à une forte densité d'intégration des composants optiques.

En dépit de ces atouts, le silicium présente plusieurs inconvénients intrinsèques pour la photonique, à savoir principalement une structure de bandes électroniques indirecte et une maille cristalline centrosymétrique. Ces propriétés conduisent à une très faible efficacité du processus d'émission de photons (pour la réalisation de sources), et à l'absence d'effet électro-optique linéaire (Pockels) couramment exploité dans les systèmes de communications modernes avec le niobate de lithium ou les matériaux semiconducteurs III/V pour la réalisation de modulateurs électro-optiques rapides. Si l'on y ajoute sa transparence dans le proche infrarouge, empêchant la réalisation de photodétecteurs dans cette gamme de longueurs d'onde, il est ainsi notable que le silicium en lui-même ne présente que peu de propriétés favorables à la réalisation des trois fonctions essentielles : source/modulation/détection d'un lien optique intégré. Malgré tout, les efforts de recherche des centres académiques et industriels déployés pour étendre 
l'usage du silicium à la photonique ont été très fortement stimulés ces dernières années par des enjeux applicatifs gigantesques : l'augmentation rapide du volume de données numériques diffusées dans le monde, principalement vers/depuis les centres de données gourmands en bande passante, les changements drastiques de l'utilisation d'Internet par les consommateurs équipés très largement d'appareils mobiles (tablettes, smartphones), et le développement plus récent des objets connectés. Dans ce contexte, les inconvénients précités du silicium ont été largement compensés par les atouts d'intégration très dense de la photonique Si et de sa co-intégration possible avec l'électronique CMOS, d'autant que des solutions originales ont été proposées et développées pour la réalisation de modulateurs silicium et de photodétecteurs Ge intégrés, apportant des solutions solides et matures pour répondre aux challenges du départ. Tous ces efforts ont été développés en tenant compte des défis récents de vitesse, de consommation électrique, de flexibilité et de fiabilité pour la réalisation d'émetteurs-récepteurs optiques pour les centres de données, de capteurs pour la détection d'agents chimiques, gazeux, biologiques... Ainsi, progressivement, le silicium est devenu une plateforme majeure pour la photonique et une réalité industrielle [2].

Depuis quelques années, des solutions combinant à la fois la photonique et l'électronique sont donc désormais disponibles pour répondre aux exigences du transfert et du traitement de l'information (figure 1a). Parallèlement, grâce à la capacité de fabrication peu coûteuse et aux volumes importants de production, des capteurs en photonique silicium sont également disponibles, notamment pour la détection précoce de maladies.

Cette tendance, sous-tendue et rendue possible par des progrès constants en termes de méthodologie de conception, de méthodes de modélisation des composants et des systèmes photoniques, et de maîtrise des spécificités de la fabrication des structures et circuits optiques (motifs en forme de courbes, règles de conception différentes de celle de l'électronique, etc.), a débouché plus récemment sur un panel d'applications possibles allant au-delà du champ initial des interconnexions optiques point à point (figure 1b) : systèmes de routage en longueurs d'onde des signaux et de parallélisations massives par multiplexage en longueurs d'onde (WDM), traitement tout optique des signaux, nouvelles applications variées telles que l'intégration de gyroscopes optiques, d'amplificateurs et d'oscillateurs intégrés, le développement de LIDAR, de spectroscopes optiques, l'intégration de fonctions photoniques pour l'optique quantique sur puce, ou la réalisation de circuits photoniques hyperfréquences et opto-hyperfréquences (radio sur guide, oscillateurs optoélectroniques).

Dans ce contexte très riche et rapidement évolutif, la figure 2 présente une vue non exhaustive de structures photoniques silicium actuellement disponibles incluant des guides d'onde rubans, des guides à base de cristaux photoniques, des structures sub-longueurs d'onde ou à fente, des modulateurs silicium à électro-réfraction basés sur le principe de la déplétion de porteurs et à électro-absorption dans des structures à multipuits quantiques (a) Photonique Si : aujourd’hui

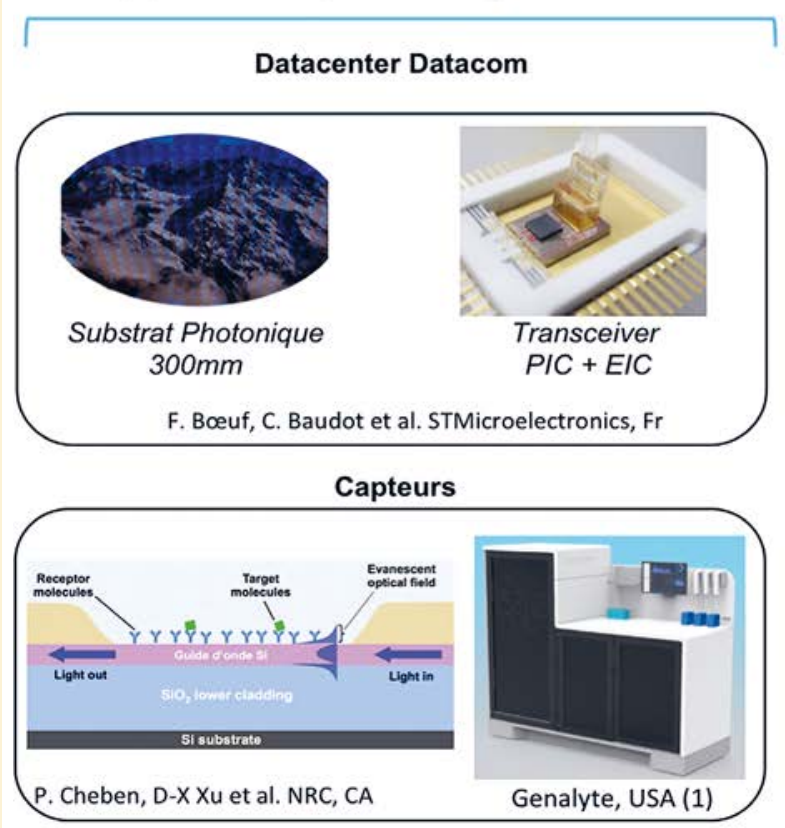

(b) Photonique Si : Demain

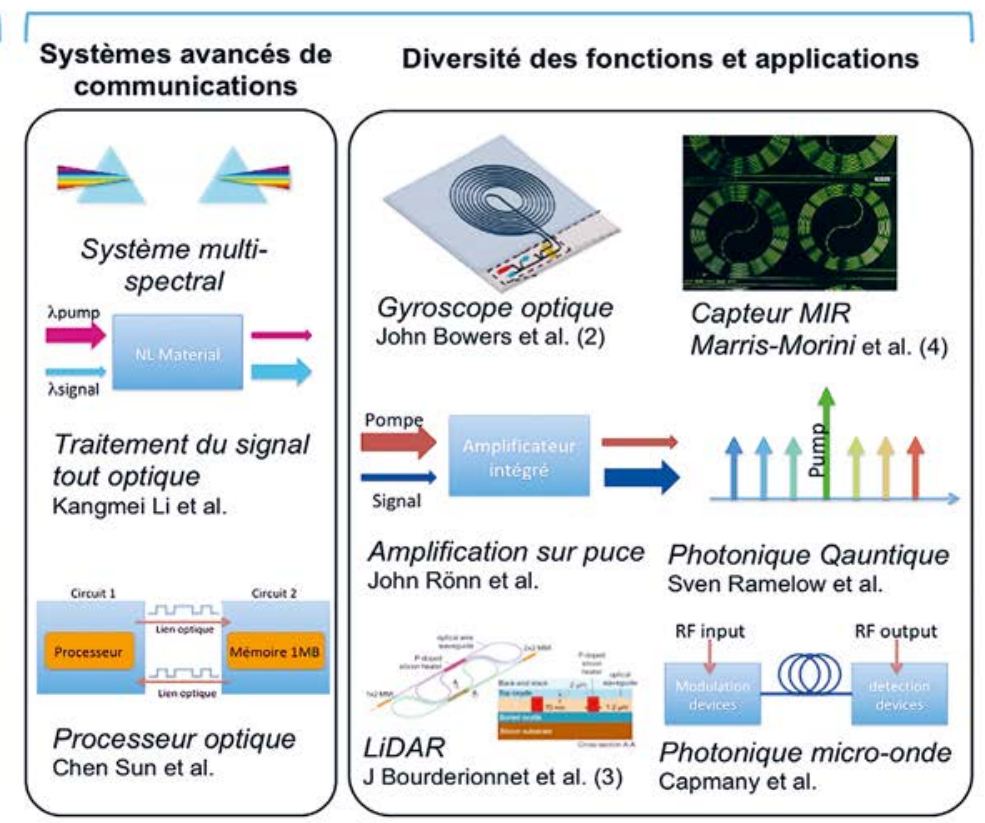

Figure 1. Évolution de la photonique silicium. (a) Aujourd'hui la photonique silicium adresse principalement deux marchés : les communications optiques inter et intra-puce rapides, notamment pour les centres de données, et la réalisation de capteurs pour la détection biologique. (b) En perspectives à court et moyen termes, de nouveaux domaines applicatifs s'offrent à la photonique silicium, comme la photonique quantique, la métrologie sur puce, et la spectroscopie d'absorption dans le moyen infrarouge, notamment pour des applications biologiques. (1) www.genalyte.com ; (2) S. Srinivasan et al., Opt. Express 22, 24988 (2014) ; (3) A. Martin et al., J. Lightwave Technology 36, 4640 (2018) ; (4) D. Marris-Morini et al., SPIE Photonics West, Jan 2018, San Francisco, United States, https://hal.archives-ouvertes.fr/hal-01798825/ 
Ge/SiGe exploitant l'effet Stark confiné quantiquement [3], et des détecteurs germanium intégrés [4].

À titre d'exemple, des caractéristiques de modulation OOK (on-offkeying) remarquables ont déjà été obtenues avec des performances proches des modulateurs niobate de lithium, une référence dans les systèmes télécoms. Des transmissions de données à des débits supérieurs à 40 Gbps ont ainsiété démontrées avec un taux d'extinction supérieur à 8 $\mathrm{dB}$ et des pertes $\mathrm{d}$ 'insertion inférieures à $5 \mathrm{~dB}$. Des efficacités de modulation, caractérisées par le produit de la tension appliquée et de la longueur du modulateur pour atteindre un déphasage de $\pi(V \pi L \pi)$, atteignent $1-2 \mathrm{~V} \mathrm{~cm}$ pour les effets à déplétion de porteurs et sont inférieures à $1 \mathrm{~V} \mathrm{~cm}$ pour des modulateurs à accumulation de porteurs à base de capacités MOS. Le deuxième exemple porte sur la démonstration de photodétecteurs intégrés en germanium dont les bandes passantes atteignent des fréquences supérieures à $100 \mathrm{GHz}$, des sensibilités de plus de $1 \mathrm{~A} / \mathrm{W}$ dans le proche infrarouge et des courants d'obscurité inférieurs au nano-ampère. Ces composants ont démontré d'excellentes caractéristiques permettant aujourd'hui de proposer des circuits plus complexes. De plus, en raison de l'impact considérable de la photonique silicium, la plupart de ces composants ont été transférés dans des fonderies industrielles accessibles via des services de fabrication multi-projets: CEA/Leti, IMEC, AIMPhotonics, IME..., rendant accessible une technologie à l'état de l'art et à fort potentiel applicatif.

Cependant, parmi tous les dispositifs actuellement disponibles, la source optique sur silicium reste un défi majeur. De nombreux travaux ont été menés pour forcer l'émission de photons et contourner l'inconvénient de la structure de bande indirecte du Si en exploitant l'effet Raman, le dopage avec des ions terres rares comme l'erbium, ou l'exploitation de nano-cristaux de silicium. La plupart de ces approches nécessitent cependant un laser externe de pompe limitant l'intérêt des solutions développées pour des applications sur puce. Une approche originale, introduite par le MIT (USA), a été d'exploiter le germanium dont les énergies des gaps direct et indirect sont très proches [5]. Ainsi, en exerçant une contrainte mécanique en tension suffisamment forte, combinée à un fort dopage du $\mathrm{Ge}$, un effet laser a été obtenu à des longueurs d'onde autour de $1550 \mathrm{~nm}$, d'abord sous pompage optique puis sous pompage électrique. Cette démonstration majeure et très prometteuse reste
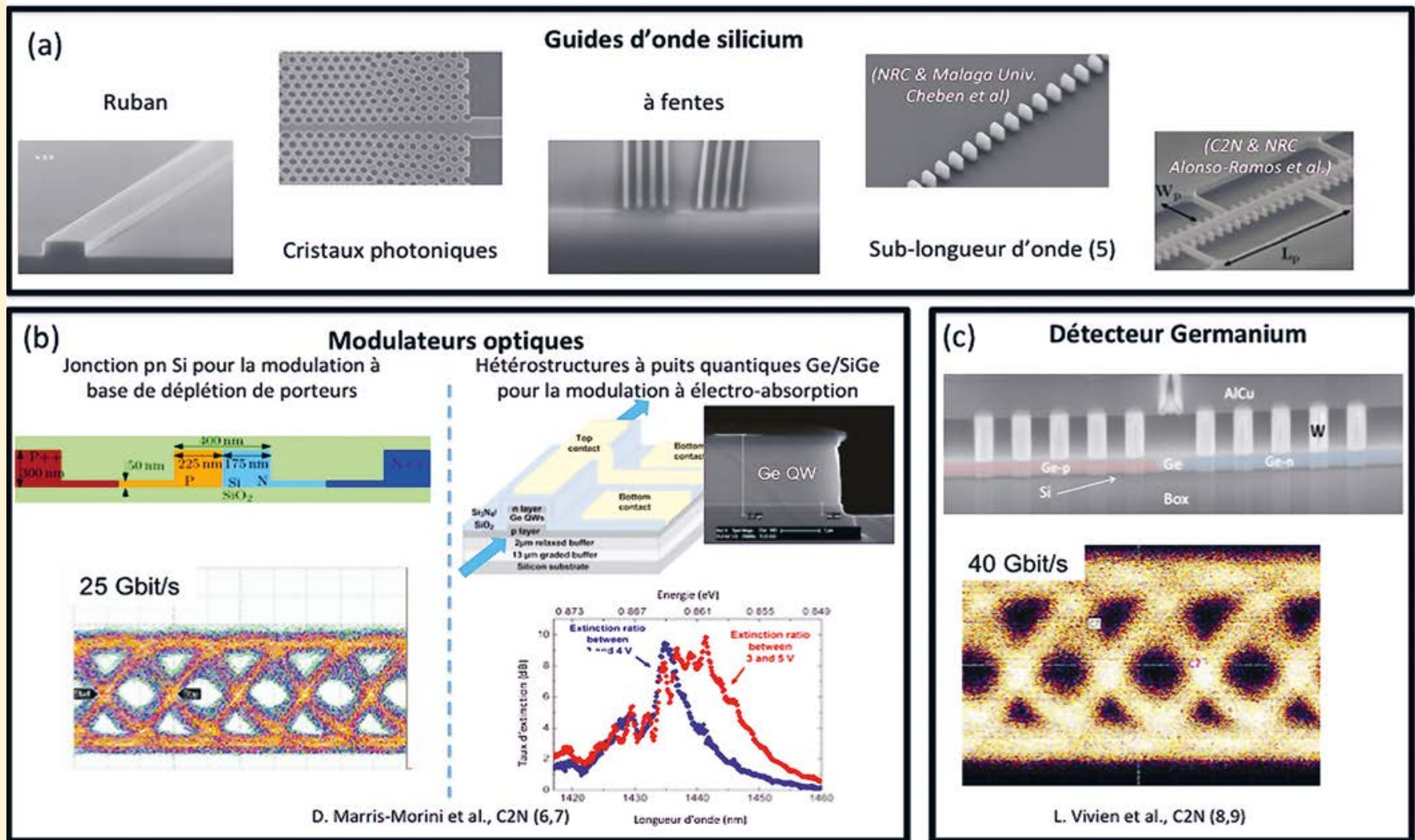

Figure 2. Composants de la photonique silicium. (a) Une variété de guides d'onde silicium a été développée incluant les guides rubans, à cristaux photoniques, à fentes et sub-longueurs d'onde. Selon les structures envisagées, il est possible de contrôler les propriétés de propagation de la lumière (dispersion, vitesse de phase et de groupe, transparence). (b) De nombreuses structures pour la modulation de phase par déplétion et accumulation de porteurs libres dans le silicium et la modulation de l'absorption dans des structures à base de germanium sur Si ont été développées. Des caractéristiques physiques remarquables en termes de fréquences de modulation, pertes d'insertion, efficacité de modulation ont été démontrées. (c) La détection de la lumière sur puce silicium se fait principalement en utilisant le germanium comme matériau absorbant. Des caractéristiques de détection proches de celles obtenues avec les semiconducteurs III-V considérés comme des références aux longueurs d'onde télécoms sont aujourd'hui atteintes. (5) P.J. Bock et al., Opt. Express 18, 20251 (2010) ; (6) D. Perez-Galacho et al., Opt. Express 25, 11217 (2017) ; (7) P. Chaisakul et al., Opt. Express 20, 3219 (2012) ; (8) L. Virot et al., Nature Communications 5, 4957 (2014) - (C) SpringerNature, figure protégée par le droit d'auteur et non couverte par la licence Creative Commons ; (9) L. Vivien et al., Opt. Express 20, 1096 (2012) 


\section{Du Composant au Système}

encore une preuve de concept et des efforts sont encore à mener pour réduire les courants de seuil et augmenter l'efficacité d'émission des structures.

Par conséquent, l'intégration d'autres matériaux sur silicium semble être une approche alternative crédible pour la réalisation de sources lasers sur silicium. En l'état actuel des efforts d'intégration hybride de matériaux émergeants (nanotubes, boîtes quantiques, etc.), les lasers les plus efficaces sur Si ont été développés à partir de semi-conducteurs III-V. En effet, les matériaux à base d'InP restent aujourd'hui le meilleur choix pour la fabrication de sources laser efficaces dans la gamme de longueurs d'onde de 1,3 à 1,55 $\mu \mathrm{m}$, voire au-delà. Pour conserver le développement photonique peu coûteux qu'apporte la plateforme silicium, deux approches ont alors été particulièrement étudiées : le collage sur structures Si de couches ou de vignettes en semiconduteurs III-V, ou l'épitaxie directe de semiconducteurs III-V sur Si. Si cette dernière approche est un sujet de recherche passionnant depuis une vingtaine d'années qui permettrait une intégration monolithique de tous les composants photoniques sur une plateforme compatible avec celle du silicium, l'hybridation par collage est cependant considérée aujourd'hui comme une solution pragmatique qui a permis plusieurs démonstrations de dispositifs hybrides III-V / Si efficaces, généralement de sources lumineuses (hauts rendements d'émission, durées de vie étendues). Cette intégration peut se faire via soit une intégration dite front-side (figure $3 a$ ), la plus courante, ou une intégration dite back-side (figure $3 b$ ) permettant de préserver les niveaux d'interconnexions métalliques compatibles avec ceux développés pour l'électronique. La plupart de ces démonstrations ont été obtenues en combinant une chaîne de fabrication silicium pour les structures photoniques (guides d'onde, modulateurs, photodétecteurs...) et une fonderie III-V conventionnelle pour la structuration des semiconducteurs III-V et la métallisation spécifique à ces matériaux (principalement de l'or pour les contacts, contaminant en Si). Un des challenges porte aujourd'hui sur l'intégration de tous les composants sur la même plateforme photonique silicium en utilisant une technologie commune. Des résultats très prometteurs ont été obtenus dans cette direction récemment avec un laser III/V sur Si fabriqué entièrement sur la plateforme Si $200 \mathrm{~mm}$ en utilisant une métallisation compatible CMOS.

Malgré toutes ces démonstrations remarquables concernant l'ensemble des composants optoélectroniques, les exigences en termes de consommation d'énergie des différentes briques de base développées pour les communications inter et intra-puces ne sont pas atteintes à ce jour. En effet, le développement de circuits complexes incluant plusieurs sources lasers, modulateurs, détecteurs et structures photoniques passives nécessite de mener une réflexion globale sur le budget énergétique des liens optiques. En particulier, il est nécessaire de diminuer les pertes optiques de toutes les structures photoniques, d'augmenter la sensibilité des récepteurs afin de réduire la puissance optique émise par les lasers, de réduire les courants de seuil des lasers, et d'augmenter l'efficacité des modulateurs optiques. Des solutions alternatives aux solutions plus conventionnelles doivent être également considérées pour répondre aux enjeux de la photonique Si et aux défis liés aux domaines applicatifs très vastes de la photonique Si. Sans être exhaustif, on peut citer les développements autour du silicium contraint pour briser la symétrie du cristal et exploiter l'effet Pockels, l'étude et l'exploitation d'hétérostructures à base de puits quantiques $\mathrm{Ge} / \mathrm{SiGe}$ pour la réalisation de modulateurs optiques compacts, l'utilisation des effets optiques non-linéaires du troisième ordre pour la réalisation de sources multi-spectrales exploitant la génération de peignes de fréquences, et toutes les études autour de l'intégration hybride de matériaux compatibles du point de vue de l'intégration technologique et présentant des propriétés physiques en rupture avec celles du silicium. Des matériaux tels que les polymères dopés, les nanomatériaux
. Micro-positionnement

- Translation

- Rotation

- Support Miroir - etc...

. Ensembles complets

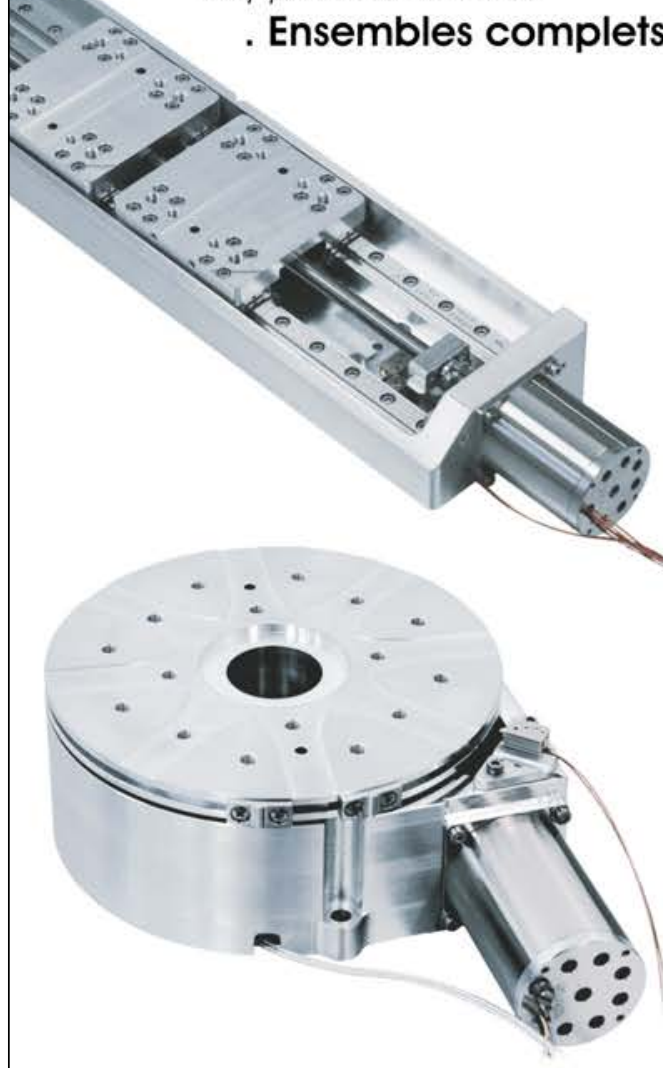

Solutions customisées ef économiques

\section{Environnements :}

\section{Standard / Spécifique}

Vide - Ultra-vide - Salle blanche

Cryo - Hautes températures

Rayonnements
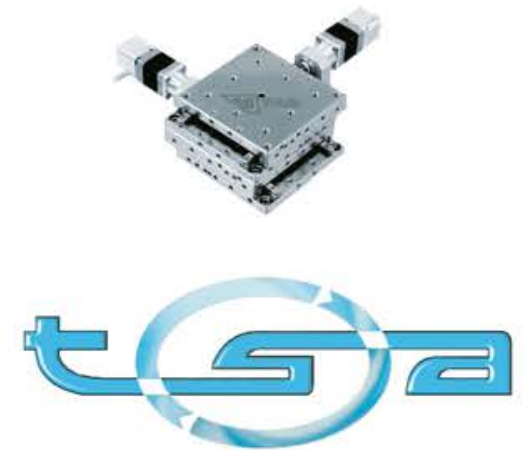

6 rue Condorcet - F-95157 Taverny Tel: + 33 (0)1.30.40.81.30 

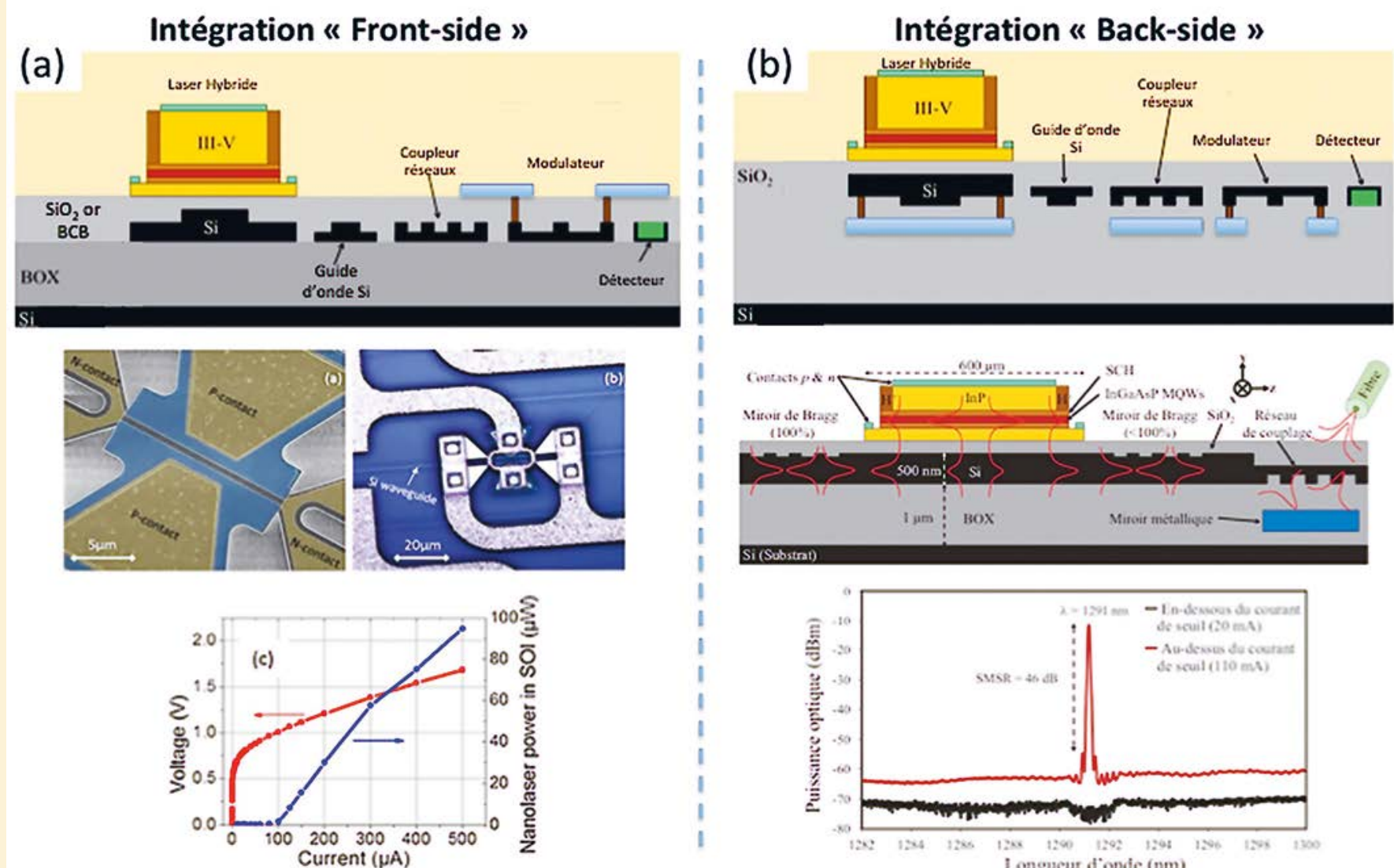

F. Raineri et al., C2N $(10,11)$

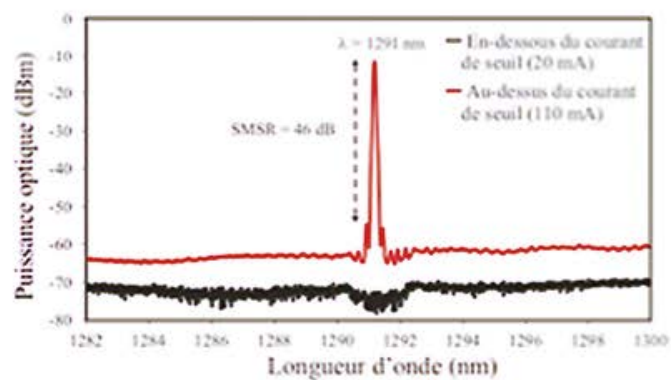

B. Szelag et al., CEA/Leti (12)

Figure 3. Intégration hybride de semiconducteurs III-V sur silicium. Les possibilités d'intégration de nouveaux matériaux comme les semiconducteurs III-V dans la plateforme silicium peut se restreindre à deux approches pour lesquelles chacune a démontré des résultats prometteurs pour l'émission laser sur Si. (a) L'intégration dite front-side où les semiconducteurs III-V sont hybridés à la surface des structures photoniques Si. Comme exemple de cette approche d'intégration couramment exploitée, une nanodiode laser pompée électriquement, intégrée sur silicium et exploitant des cristaux photoniques, a été démontrée par le $\mathrm{C} 2 \mathrm{~N}$, avec une efficacité compatible d'une intégration à grande échelle dans les futurs circuits intégrés [6]

(b) L'intégration dite back-side où les semiconducteurs III-V sont intégrés en-dessous des structures photoniques après avoir retiré le substrat de silicium et la couche de silice enterrée. Cette approche, privilégiée par les industriels, permet de garder compatibles les niveaux de métallisation de circuits existants. Une première démonstration par le CEA/Leti et STMicroelectronics a été effectuée sur plate-forme Si $200 \mathrm{~mm}[7]$. (10,11) G. Crosnier et al., Nature Photonics 11, 297 (2017) - C SpringerNature, figure protégée par le droit d'auteur et non couverte par la licence Creative Commons; (12) J. Durel, Thèse de Doctorat de la communauté Université Grenoble Alpes, 25 mai 2016, https://tel.archives-ouvertes.fr/tel-01689987/

1D (nanofils, nanotubes...), les matériaux 2D (graphène, $\mathrm{MoS}_{2} \ldots$ ) ou $0 \mathrm{D}$ (boîtes quantiques...), les verres de chalcogénure, les métaux pour exploiter des effets plasmoniques, les oxydes fonctionnels (BTO, BST, YSZ...) et les semiconducteurs III-V sont quelques exemples des nombreuses possibilités offertes par l'intégration hybride sur Si.

Pour conclure, grâce à sa transparence dans une large gamme de longueurs d'onde et à sa compatibilité avec les processus CMOS développés pour la microélectronique, la photonique silicium est apparue comme incontournable pour répondre aux nouveaux défis des circuits intégrés. Les applications visées dépassent aujourd'hui le simple domaine des communications optiques avec notamment la photonique quantique, les capteurs et la métrologie qui en font de nouveaux

défis à relever pour la photonique silicium dans les prochaines années.

\section{POUR EN SAVOIR PLUS}

[1] L. Vivien, L. Pavesi, Handbook of Silicon photonics, CRC Press, 2013

[2] F. Boeuf, S. Cremer, E. Temporiti, M. Fere, M. Shaw, C. Baudot, N. Vulliet, T. Pinguet, A. Mekis, G. Masini, H. Petiton, P. Le Maitre, M. Traldi, L. Maggi, Silicon Photonics R\&D and Manufacturing on 300-mm Wafer Platform, J. Light. Technol. 34, 286 (2016)

[3] P. Chaisakul, D. Marris-Morini,J. Frigerio, D. Chrastina, M.-S. Rouifed, S. Cecchi, P. Crozat, G. Isella, L. Vivien, Integrated germanium optical interconnects on silicon substrates", Nature Photonics 8, 482 (2014)

[4] L. Virot, P. Crozat, J.-M. Fédéli, J.-M. Hartmann, D. Marris-Morini, E. Cassan, F. Boeuf, L. Vivien, Germanium avalanche receiver for low power interconnects, Nat. Commun. 5, 4957 (2014)

[5] R.E. Camacho-Aguilera, Y. Cai, N. Patel, J.T. Bessette, M. Romagnoli, L.C. Kimerling, J. Michel, An electrically pumped germanium laser, Optics Express 20, 11316 (2012)

[6] G. Crosnier, D. Sanchez, S. Bouchoule, P. Monnier, G. Beaudoin, I. Sagnes, R. Raj, F. Raineri, Hybrid indium phosphide-on-silicon nanolaser diode, Nature Photonics 11, 297 (2017)

[7] J. Durel, B. Ben Bakir, C. Jany, S. Cremer, B. Szelag, T. Bria, V. Larrey, L. Sanchez, P. Brianceau, J.-A Dallery, R. Guiavarch, T.Card, R.Thibon, J.-E. Broquin, F. Bøuf, First Demonstration of a BackSide Integrated Heterogeneous Hybrid III-V/Si DBR Lasers for Si- Photonics Applications, 2016 IEEE International Electron Devices Meeting (IEDM) 